\title{
Novel Tune Diagnostics for the Tevatron*
}

\author{
C.Y. $\operatorname{Tan}^{\dagger}$, FNAL, P.O. Box 500, Batavia, IL 60510, USA
}

\section{Abstract}

In the Tevatron collider, protons and antiprotons share the same beam pipe. This poses a challenge in the measurement of tunes for both species simultanously because of the possibility of signal contamination from the other species. The tune of each bunch is also very different because of beam-beam effects from parasitic crossing points. This means that the tune diagnostics must be able to differentiate between protons and anti-protons, it also has to measure tunes from each bunch. There are three different tune pickups used in the Tevatron: 1.7 GHz Schottky pickups, 21.4 MHz Schottky pickups and baseband pickups. These devices will be discussed in detail in this paper.

\section{INTRODUCTION}

The Tevatron is a single beam pipe machine which collides protons and anti-protons at two experiments CDF and D0. Presently for high energy physics (HEP), the Tevatron collides 36 bunches of protons on 36 bunches of antiprotons. The 36 bunches of each species are divided into 3 trains of 12 bunches each with each bunch in each train separated by about 396 ns. Presently, the beam current of protons is approximately 10 times larger than the antiprotons.

In order to keep these two species apart in the beam pipe at locations other than the experiments, electrostatic separators are used to distort the orbits so that each species travels on mirrored orbits. The two species are separated everywhere except at the two experiments. The remaining $(36 \times 2)-2=70$ points in the ring where the two species get close transversely but do not collide are called the parasitic crossing points.

One consequence[1] of partitioning the bunches into 3 trains, is that the bunches at the end of each train experiences a significantly different amount of beam-beam kicks from the opposite species than the bunches in the middle. This causes the betatron tunes of the first and last bunch of each train to have very different tunes compared to the bunches in the middle of the train. See Figure 1. Therefore, good knowledge of tunes is essential for Tevatron operations.

The ideal tune measurement system for the Tevatron must not only be able to differentiate between protons and anti-protons, it needs to be parasitic, bunch by bunch and be able to measures tunes at injection, up the ramp and through the squeeze. Its electronics or software must be

\footnotetext{
* Supported by the United States Department of Energy under Contract No. DE-AC02-76CH03000 and the U.S. LHC Acelerator Research Program (US LARP).

†cytan@fnal.gov
}

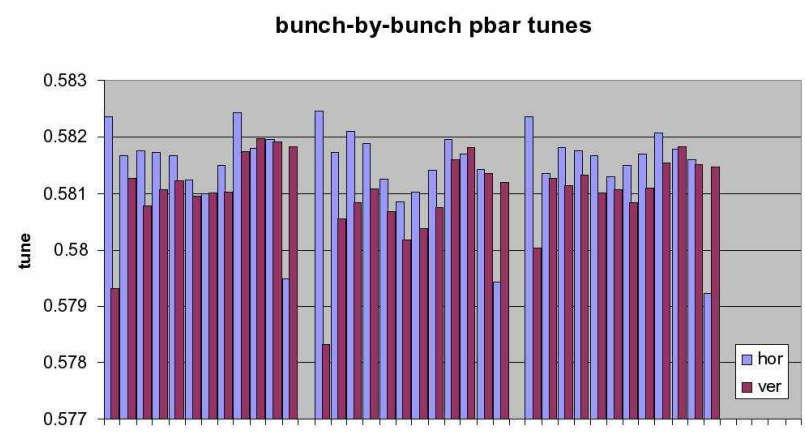

Figure 1: The individual pbar tunes at collisions measured by the $1.7 \mathrm{GHz}$ Schottky shows (a) first/last bunch of each train has a significantly different vertical/horizontal tune than those in the middle of the train (b) 3-fold symmetry. (Courtesy of A. Jansson)

able to measure tunes to $10^{-4}$ and return readings at $1 \mathrm{~Hz}$. Unfortunately none of the present tune measurement systems satisfy all these requirements.

For the pickup, the necessary properties which are required for differentiating between protons and anti-protons and for individual tunes to be measured are as follows:

- Pickup directivity. Pickups are designed to have directivity. For example, striplines have $\sim 30 \mathrm{~dB}$ of directivity.

- Bandwidth The bandwidth of the pickup must be high enough $\sim 100 \mathrm{MHz}$ so that bunch signals are distinct and can be gated.

- Choice of location. The pickups are placed in the Tevatron so that the protons and anti-protons are well separated in time at both injection and at collisions because cogging causes the relative positions of the protons and anti-protons to move by about 62 buckets or $1.2 \mu \mathrm{s}$ at collisions.

Figure 2 shows the output from the anti-proton end of a stripline pickup at D48 at collisions. Things to notice are that the position of the stripline had been chosen so that there is clear temporal separation between the two species; there is some proton signal before the RF switch despite the directivity of the stripline and finally with the RF switch, only the anti-proton signals are allowed into the electronics.

\section{The Tune Measurement Devices}

There are three types of tune measurement devices used in the Tevatron. Each covering a different band of the tune spectrum and thus exploring a different physics regime: 


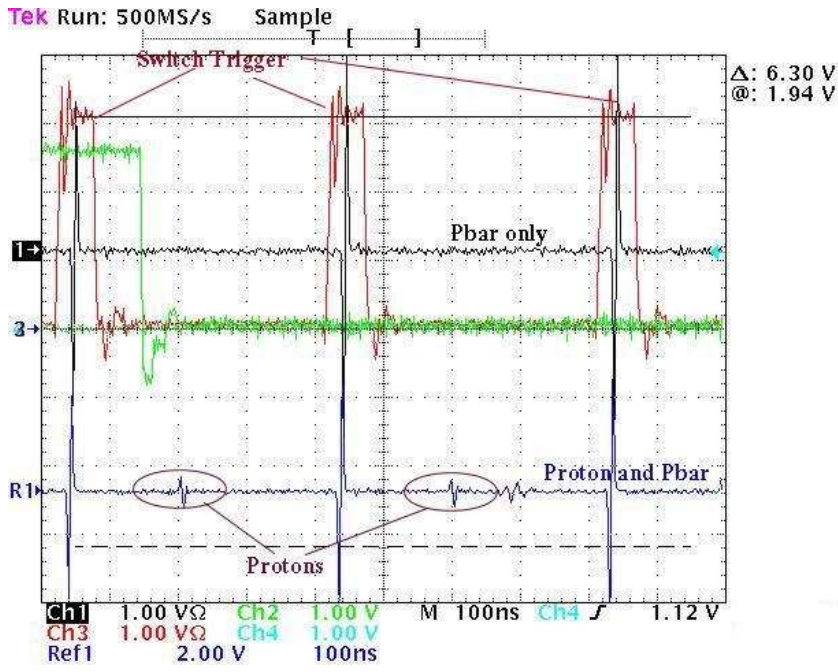

Figure 2: The blue trace is the signal from the anti-proton end of the stripline which shows signals from both protons and anti-protons. The red trace is the switch trigger which opens the gate for the anti-proton signal. The black trace is the anti-proton signal after the RF switch. The green trace is the revolution marker.

- 21.4 MHz Schottky detector. This detector was installed in 1989 and is the oldest of all the tune measuring devices used in the Tevatron. This detector is the default standard for measuring proton tunes despite its limitations because historically it was the only detector available. This is a resonant structure that has some directivity. Despite the directivity, the proton signals contaminate the anti-proton signals and so in order to see the anti-proton tunes, the anti-protons have to be excited. Furthermore, because of its high $Q$, it is not possible to gate individual bunches at the detector. However, by gating the kick instead, individual antiproton tunes can be measured. This gated kick method cannot be used for the protons because the unkicked signal is already strong enough to produce a tune spectrum.

- $1.7 \mathrm{GHz}$ Schottky detector. This detector was installed in 2002. It was a breakthrough detector for the Tevatron because it was designed to be able to see anti-proton tunes without excitation and to measure individual bunch tunes. This detector is a slow wave waveguide structure which has directivity and designed with a low $Q \sim 20$ so that individual proton and anti-proton tunes can be gated and measured.

- 3D-BBQ (Direct Diode Detector Baseband tune). This is the newest system in the Tevatron and was installed this year. The novelty of this system lies in the electronics which at its heart is a diode detector. This system uses a stripline pickup so that the proton and anti-proton signals can be gated. Presently, it is an $R \& D$ project funded under US LARP. This system is presently used to cross check the tunes measured by both the $21.4 \mathrm{MHz}$ and the $1.7 \mathrm{GHz}$ detectors.

\subsection{MHZ SCHOTTKY DETECTOR}

These detectors [2] are $1 \mathrm{~m}$ in length with a square cross section shown in Figure 3. The stripline consists of two copper bars which can be moved by stepper motors to change the sensitivity. Its resonant frequency can also be tuned by using the variable capacitor $C_{v}$. Presently these detectors are tuned to resonate around $21.4 \mathrm{MHz}$ with a loaded $Q_{L}=370$.

Four of these detectors have been built to allow the user to look at the horizontal and vertical tunes of both the protons and anti-protons. However in practice, only the proton tunes are monitored during high energy physics (HEP) because the proton tunes are usually clear and unambiguous. The anti-protons tunes are not normally measured or monitored because a lot of the proton signal still leaks through which swamps the anti-proton signals. In order to overcome this problem, the anti-protons have to be gently kicked or tickled in order to bring them out.[5]

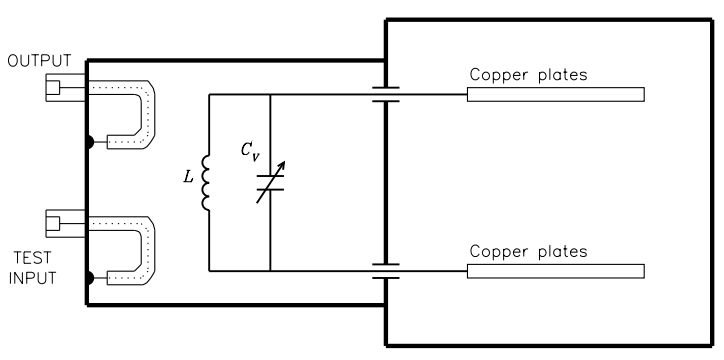

Figure 3: Transverse geometry of the Schottky pickup reproduced from [2]. (Courtesy of B. Fellenz)

Figure 4 shows the results when a vertical stripline kicker is gated to tickle anti-proton bunch 24 with bandwidth limited white noise. The tickle on/off pictures shows that tickling brings the tune of this bunch out of the noise floor. Clearly, before the tickle, enough of the proton signal has leaked through into the anti-proton channel of the detector. After the tickle is applied, the anti-proton vertical tune rises above the noise floor and is clearly seen.

Despite being able to see anti-protons tunes with tickling, the spectrum contains lines which are not easily explained. Furthermore, the problem with tickling is that it blows up the emittance of the beam and causes poor lifetime of the anti-protons. This limits the use of the 21.4 MHz Schottky to measuring anti-protons tunes at the end of HEP.

Tune Tracker and Tune Fitter with $21.4 \mathrm{MHz}$ Schottky

The Tevatron tune tracker[3] is based on the phase locked loop technique that was pioneered at both RHIC and CERN[4]. The tune tune tracker uses the 

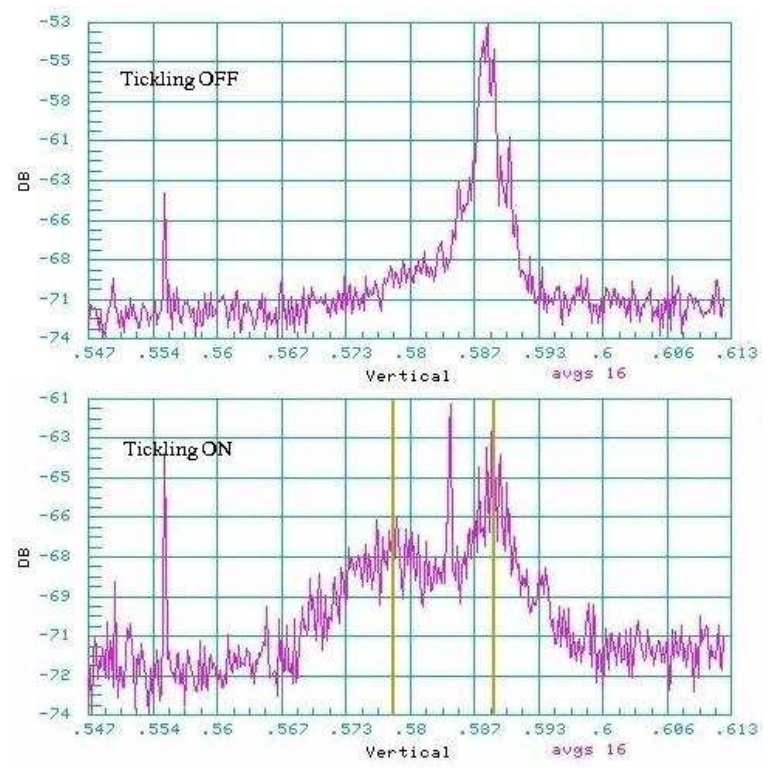

Figure 4: Anti-proton tune of bunch 24 is 0.5783 with gated tickling $\mathrm{ON}$ and $\mathrm{OFF}$ at collisions. The proton tune is at 0.5888 .

21.4 MHz Schottky for measuring the proton tunes with accuracy better than $10^{-4}$. This method requires beam excitation but has been shown not to cause any measurable emittance growth at injection. The present tune tracker is able to track the tunes up the ramp and through the squeeze. It is also used to measure chromaticities by tracking the tunes when the RF frequency is ramped. An example of measuring chromaticity with this technique is shown in Figure 5.

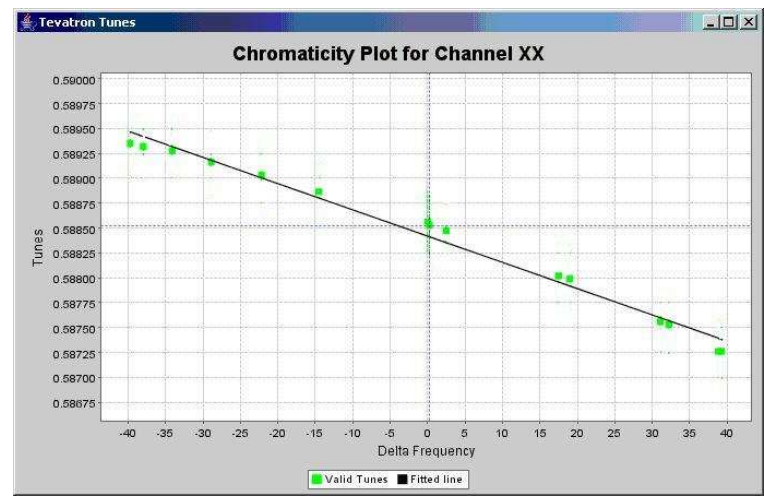

Figure 5: Horizontal chromaticity measurement by changing the RF frequency and tracking the tune. The proton chromaticity measured here is $3.7 \pm 0.2$.

The tune fitter is a software programme written by P. Lebrun[7] which uses sophisticated tune fitting techniques to find the peaks of the tunes. This method, unlike the tune tracker is parasitic.

The advantage of the tune tracker over the tune fitter is that the tune tracker returns tune data to the control system at $10 \mathrm{~Hz}$ while the tune fitter returns data at $0.1 \mathrm{~Hz}$.

\subsection{GHZ SCHOTTKY DETECTOR}

The $1.7 \mathrm{GHz}$ Schottky Detector[6] is a $1 \mathrm{~m}$ long slow wave waveguide. Its $Q \sim 20$ was chosen so that gating can be used to pick out individual bunch signals.

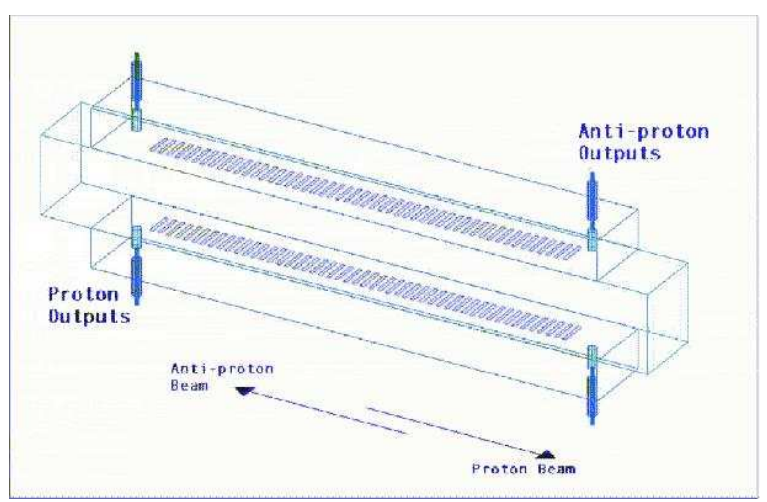

Figure 6: The $1.7 \mathrm{GHz}$ slotted waveguide is $109 \mathrm{~mm} \times$ $79 \mathrm{~mm}$ in aperture. (Courtesy of R. Pasquinelli)

The physics potential of this detector became fully realized when sophisticated curve fitting software was written which could automatically measure various beam parameters like tune, chromaticity, momentum spread for each bunch.[7] For example, during HEP shown in Figure 7, the evolution of the proton and anti-proton tunes are clearly seen when the beam-beam effects become smaller as the beam current decreases and emittance increases during the HEP store. The anti-proton tunes are shifted to lower tune by by about 0.01 in both planes.

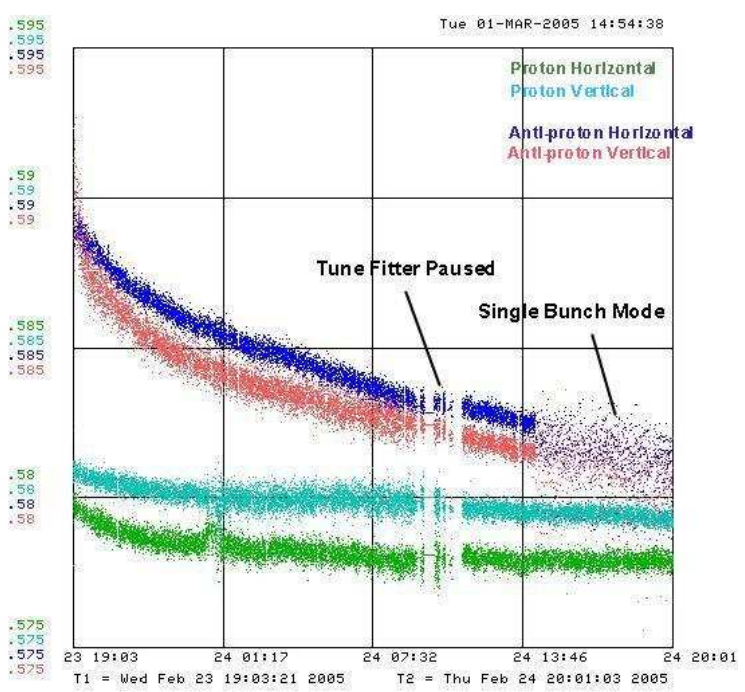

Figure 7: The beam-beam effect on tunes during a HEP store. As the emittance grows and proton beam current decreases during the store, the anti-proton tunes get shifted to lower tunes. (Courtesy of A. Jansson) 
The tune fitter uses 2 dual channel vector signal analyzers to take data from each plane for each species. Tune fitting is computationally intensive and it takes 20 minutes to cycle through the 36 bunches of each species. The rms error of the fit when all 36 bunches are averaged is $\sim 10^{-4}$ and $\sim 4 \times 10^{-4}$ for a single bunch.

Figure 8 shows the $3 \mathrm{~dB}$ width of the tune is about 0.025. The shape of the tune has contributions not only from chromaticity and momentum spread, it also constains the weighted sum of the vertical and horizontal Schottky signals. These signals sum in such a manner as to produce a centre of mass tune that is not the coupled tune but tunes which closely approximate the decoupled tune.[8].

At present the $1.7 \mathrm{GHz}$ Schottky is only useful at collisions because the coherent motion of the beam up the ramp produces signals which are not fully understood.

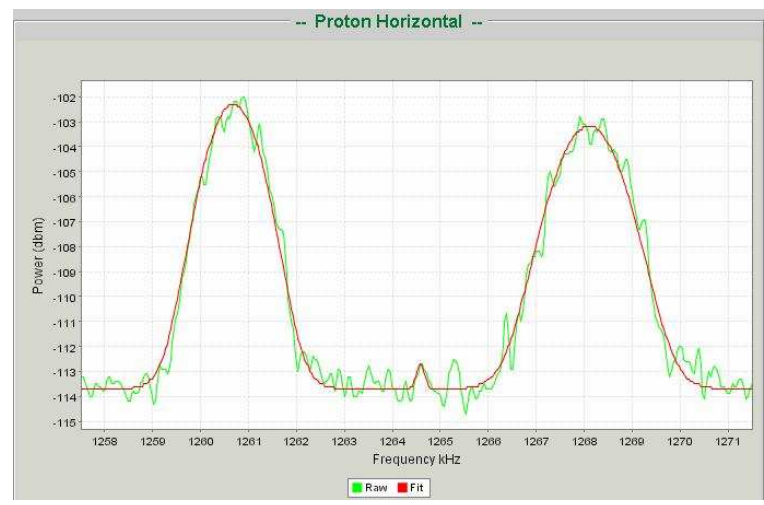

Figure 8: The lower and upper betatron tune sidebands. Notice that the span is $12 \mathrm{kHz}$ compared to the span of $2.5 \mathrm{kHz}$ used by the $21.4 \mathrm{MHz}$ Schottky detector shown in Figure 4. The difference in width and height between the sidebands comes from chromaticity. The average width is the momentum spread. (Courtesy of A. Jansson)

\section{D-BBQ}

The 3D-BBQ is invented by M. Gasior and has been installed in the Tevatron as part of the US LARP collaboration. The 3D-BBQ is an LHC development and has been evaluated and installed not only at Fermilab, but also in the CERN SPS and RHIC.[9] The 3D-BBQ block diagram is shown in Figure 9. The pickup used by the 3D-BBQ can be any non-resonant pickup and at the Tevatron, it is a $0.5 \mathrm{~m}$ stripline. The key to the 3D-BBQ electronics is the diode detector and BPF (bandpass filter). The diode detector can be thought of as an envelope detector or AM demodulator. The output of the diode detector in the frequency domain can be thought of as the folding to base band all the betatron tunes around multiples of the revolution harmonic. The advantage of doing so is that unlike the $21.4 \mathrm{MHz}$ and $1.7 \mathrm{GHz}$ Schottky systems which measure one betatron tune out of thousands, the 3D-BBQ betatron signal is enhanced by the superposition of all these betatron lines.
Furthermore, like the $1.7 \mathrm{GHz}$ Schottky, the 3D-BBQ can see anti-protons without beam excitation.

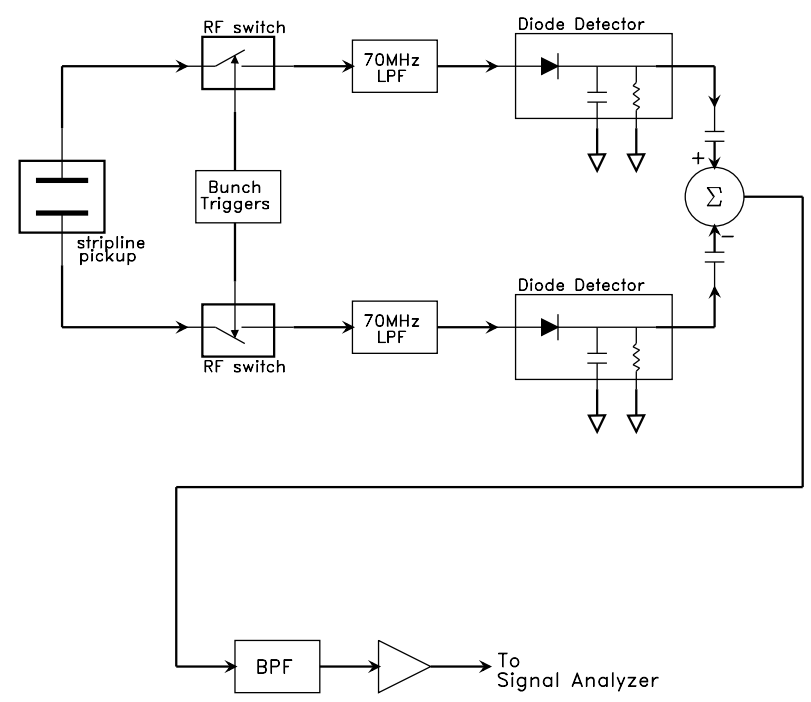

Figure 9: The 3D-BBQ with RF switch.

The BPF frequency response is shown in Figure 10. The BPF has been tuned so that its notch is at the first Tevatron revolution harmonic. This filter attenuates the revolution harmonic by $90 \mathrm{~dB}$ in order to prevent the downstream amplifier from saturating.
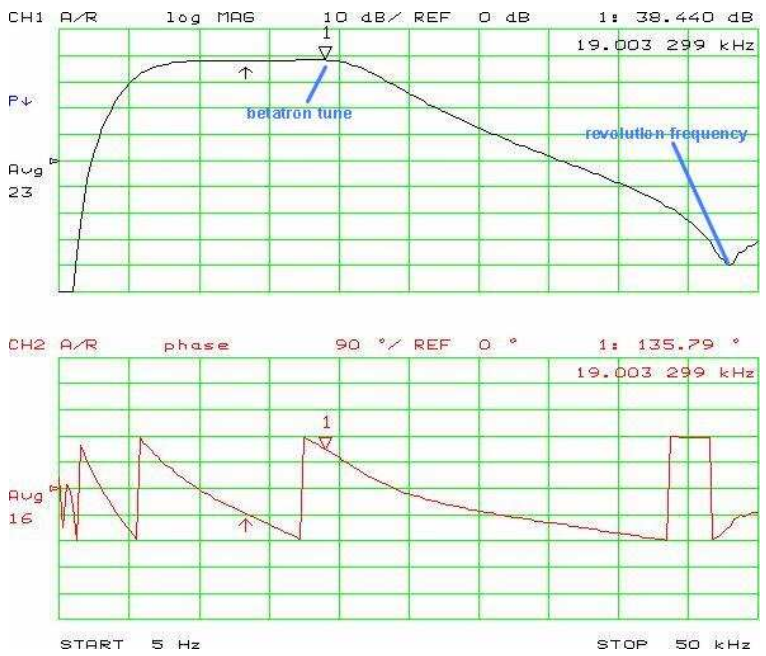

Figure 10: The bandpass filter used to notch out the revolution harmonic. The blue pointers in the figure show where the betatron tune and the revolution frequency are in the Tevatron.

The results of gating the anti-proton signal is shown in Figure 11. All 36 protons and anti-proton bunches 1 to 4, 13 to 16 and 25 to 28 are shown here. Things to notice are that the anti-proton tunes are shifted w.r.t. the protons and that there are sharp $60 \mathrm{~Hz}$ lines present in both spectra.[10] 


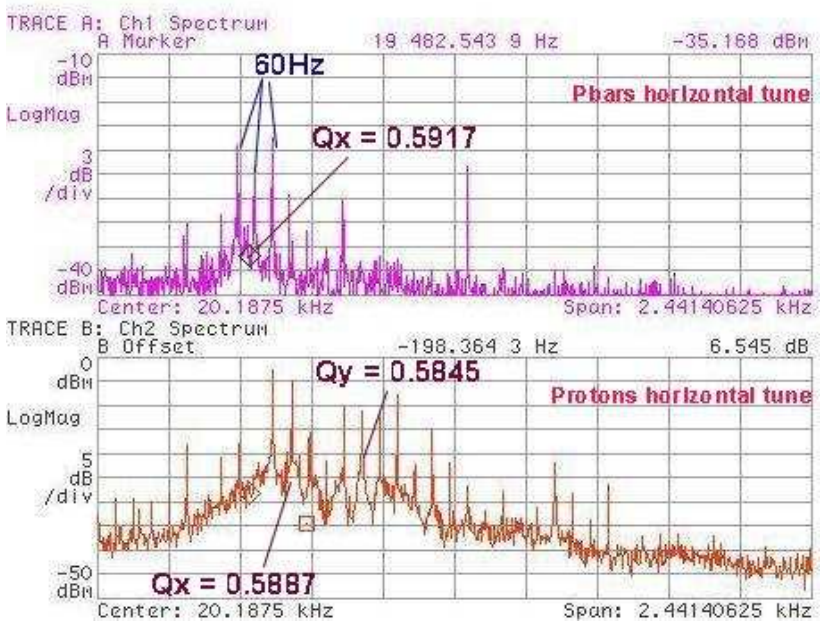

Figure 11: Shown here are the horizontal tunes of the pbars and protons at injection. Note that the quoted fractional tunes are above the $\frac{1}{2}$-integer while the measured is below the $\frac{1}{2}$-integer.

The sensitivity of the 3D-BBQ versus the $21.4 \mathrm{MHz}$ Schottky is shown in Figure 12. The 3D-BBQ is between 4 to $6 \mathrm{~dB}$ more sensitive than the $21.4 \mathrm{MHz}$ resonant detector. With the new sensitivity of the 3D-BBQ, $60 \mathrm{~Hz}$ lines in the betatron spectrum during a HEP store was seen for the first time. These lines from mains are also seen at RHIC and at the SPS (which is $50 \mathrm{~Hz}$ ).

Tune Measured by BBO

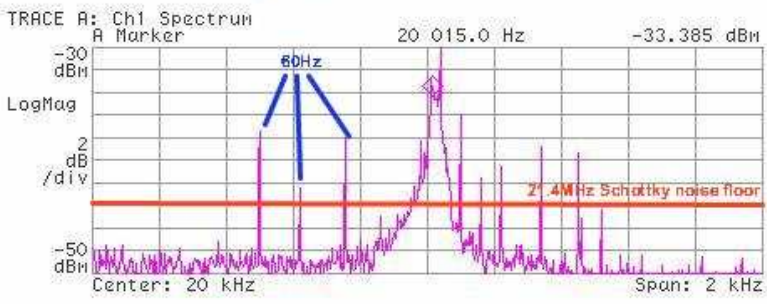

Tune Measured by 21. MHz Schottky

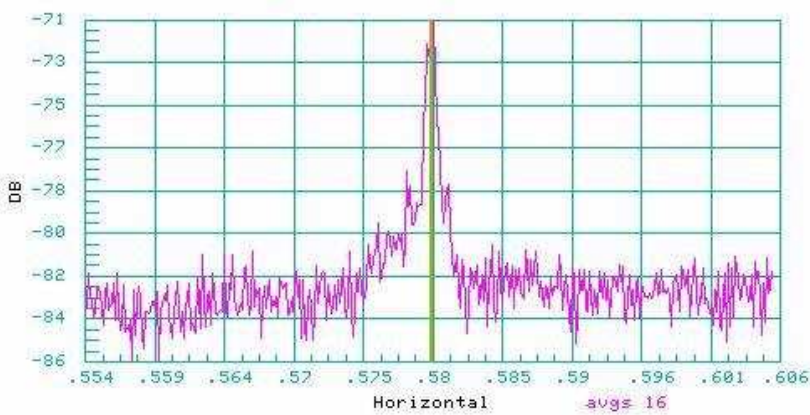

Figure 12: Comparing the sensitivity of the $21.4 \mathrm{MHz}$ Schottky and the 3D-BBQ. The red line in the BBQ spectrum is the noise floor of the $21.4 \mathrm{MHz}$ Schottky. The 3DBBQ also sees $60 \mathrm{~Hz}$ lines.
The 3D-BBQ is still an R\&D project here at the Tevatron. The measured betatron spectrum still needs to be understood. However, the low cost, sensitivity and the ability to measure individual bunch tunes makes it an important tool for the future.

\section{CONCLUSION}

The three different types of tune measurement devices used in the Tevatron provide complementary physics information. The $21.4 \mathrm{MHz}$ and the $1.7 \mathrm{GHz}$ systems are operational and provide useful physics data. The 3D-BBQ system is still in development and will be deployed in the near future.

\section{ACKNOWLEDGEMENTS}

The author wishes to thank M. Gasior (CERN) for building the 3D-BBQ system for Fermilab and P. Lebrun (FNAL) for writing the chromaticity measurement programme used by the tune tracker. The author also wishes to thank the following people at Fermilab for generously supplying the figures used in this paper: B. Fellenz, A. Jansson and R. Pasquinelli.

\section{REFERENCES}

[1] T. Sen, et al, "Beam-Beam Effects at the Fermilab Tevatron: Theory.” Phys.Rev.ST Accel.Beams 7:041001,2004.

[2] D. Martin, et al, "A Resonant Beam Detector for Tevatron Tune Monitoring”, Accelerator Science and Technology Proceedings, vol 3 1486-1488, 1989.

[3] C.Y. Tan, "The Tevatron Tune Tracker PLL - Theory, Implementation and Measurements", Fermilab Technical Memo, TM-2275, 2004.

[4] P. Cameron et al, "RHIC Third Generation PLL Tune System", Proceedings of the Particle Accelerator Conference 2003, p. 524-526.

[5] C.Y. Tan and X.L. Zhang, "Tune Measurement Methods of the Tevatron", Proceedings of the Particle Accelerator Conference 2003, p. 2703-2705.

[6] R. Pasquinelli, “A $1.7 \mathrm{GHz}$ Waveguide Schottky Detector System", Proceedings of the Particle Accelerator Conference 2003, p. 3068-3070.

[7] P. Lebrun, "Tevatron Tune Fitters, $1.7 \mathrm{GHz} \& 21.4 \mathrm{MHz}$ : Sept 04 Status Report", Beams Document 1339-v2, http://beamdocs.fnal.gov, 2004.

[8] A. Jansson, "Effect of Coupling on Tevatron 1.7 GHz Schottky Tunes", Beams Document 1576-v2, http://beamdocs.fnal.gov, 2005.

[9] M. Gasior \& R. Jones, "High Sensitivity Tune Measurement by Direct Diode Detection", 7th European Workshop on Beam Diagnostics and Instrumentation for Particle Accelerators (DIPAC), 2005.

[10] P. Cameron et al, "The Effects and Possible Origins of Mains Ripple in the Vicinity of the Betatron Spectrum", 7th European Workshop on Beam Diagnostics and Instrumentation for Particle Accelerators (DIPAC), 2005. 